\title{
Luminescent coupling in planar opto-electronic devices
}

\author{
Matthew Wilkins, ${ }^{1, a)}$ Christopher E. Valdivia, ${ }^{1}$ Ahmed M. Gabr, ${ }^{1}$ Denis Masson, ${ }^{2}$ \\ Simon Fafard, ${ }^{2}$ and Karin Hinzer $^{1}$ \\ ${ }^{1}$ School of Electrical Engineering and Computer Science, University of Ottawa, 25 Templeton St., \\ Ottawa, Ontario K1N 6N5, Canada \\ ${ }^{2}$ Azastra Opto, Inc., Suite M50-IPF-206, 1200 Montreal Rd, Ottawa, Ontario K1A OR6, Canada
}

(Received 16 July 2015; accepted 27 September 2015; published online 12 October 2015)

\begin{abstract}
Effects of luminescent coupling are observed in monolithic $5 \mathrm{~V}$, five-junction GaAs phototransducers. Power conversion efficiency was measured at $61.6 \% \pm 3 \%$ under the continuous, monochromatic illumination for which they were designed. Modeling shows that photon recycling can account for up to $350 \mathrm{mV}$ of photovoltage in these devices. Drift-diffusion based simulations including a luminescent coupling term in the continuity equation show a broadening of the internal quantum efficiency curve which agrees well with experimental measurements. Luminescent coupling is shown to expand the spectral bandwidth of the phototransducer by a factor of at least 3.5 for devices with three or more junctions, even in cases where multiple absorption/emission events are required to transfer excess carriers into the limiting junction. We present a detailed description of the novel luminescent coupling modeling technique used to predict these performance enhancements. @ 2015 AIP Publishing LLC. [http://dx.doi.org/10.1063/1.4932660]
\end{abstract}

\section{INTRODUCTION}

In recent years as the quality of III-V photovoltaic materials, such as $\mathrm{GaAs}$ and $\mathrm{Ga}_{0.5} \mathrm{In}_{0.5} \mathrm{P}$, has improved, the effects of photon recycling and luminescent coupling have become increasingly significant in the design of very-high efficiency photovoltaic devices. Photon recycling refers to emission and re-absorption, typically within a single material layer or junction, and can yield an increase in open-circuit voltage of single- and multi-junction devices. ${ }^{1}$ Luminescent coupling refers to emissions of photons from one location in a device which is re-absorbed, possibly in a different device region, and has been shown to reduce spectral sensitivity of multijunction solar cells. $^{2}$

Techniques have been developed to quantify the degree of coupling between junctions in a multi-junction solar cell, ${ }^{3}$ and to model the effects using lumped- or distributedparameter models. ${ }^{4}$ Létay et al. have also modelled photon recycling effects in single junctions within a drift-diffusion simulator, using an iterative approach which involves running the simulator repeatedly with successive approximations of the coupled photocurrent. ${ }^{5-8}$ Luminescent coupling has also been incorporated into simulations of light-emitting diodes. ${ }^{9}$

In this work, we present a photovoltaic device with five monolithically integrated GaAs junctions displaying strong effects attributed to luminescent coupling. In typical multijunction solar cells, each junction has a different band gap, and therefore, coupling proceeds in only one direction (from high to low band gap materials). However, in this device, coupling must be considered over all junction permutations. We analyze the device using a newly developed, two-step technique which incorporates the modeling of luminescent coupling $^{10,11}$ into drift-diffusion based simulations ${ }^{12,13}$ in a

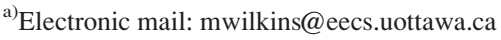

more direct way than that used by the previous methods. The details of the modeling theory are outlined in the Appendix.

The device studied here is a phototransducer designed and manufactured by Azastra Opto, Inc. for use as a receiver in a power-over-fiber application. The term "phototransducer" is used to clearly distinguish them from solar cells; these devices have a structure similar to multi-junction solar cells, but all junctions are composed of GaAs of varying thicknesses (Fig. 1(a)). They are intended to be coupled with a laser of up to $5 \mathrm{~W}$ at a single wavelength, $835 \mathrm{~nm}$. To date, we have measured a power conversion efficiency of $61.6 \% \pm 3 \%$ and open-circuit voltage of $5.92 \mathrm{~V}$ under $\sim 1.3 \mathrm{~W}$ singlewavelength illumination. ${ }^{14}$ Short-circuit current density was $4.5 \mathrm{~A} / \mathrm{cm}^{2}$. In the absence of luminescent coupling, the overall device current is limited by the lowest photocurrent in any of the five junctions, and so the device is sensitive to the incident wavelength and to any variations in layer thicknesses. At shorter-than-nominal wavelengths, light is absorbed strongly in the upper junctions (due to the higher absorptivity) and the lower junctions become limiting, while at longer wavelengths the upper junctions are limiting the overall device current.

In junctions able to produce higher photocurrent than the limiting one, excess photogenerated carriers will recombine either through a non-radiative or a radiative mechanism. The radiated photons may thereafter be re-absorbed within the same junction (photon recycling), a different junction (luminescent coupling), or exit the device (photoluminescence). For good-quality materials with sufficiently high internal radiative efficiency (fraction of radiative to total recombination), photon recycling leads to an accumulation of carriers and an increase in photovoltage, while luminescent coupling can help to redistribute photocurrent between the junctions more optimally, reducing the wavelength sensitivity.

The multi-junction photovoltaic device discussed here provides an excellent demonstration of photon recycling and luminescent coupling due to its limited set of relatively 
(a)

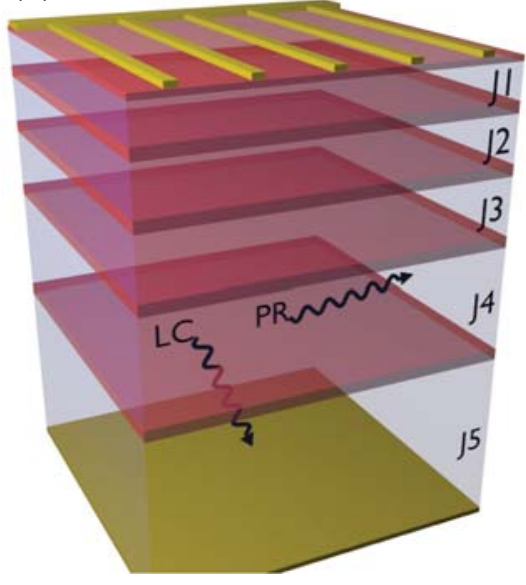

(b)

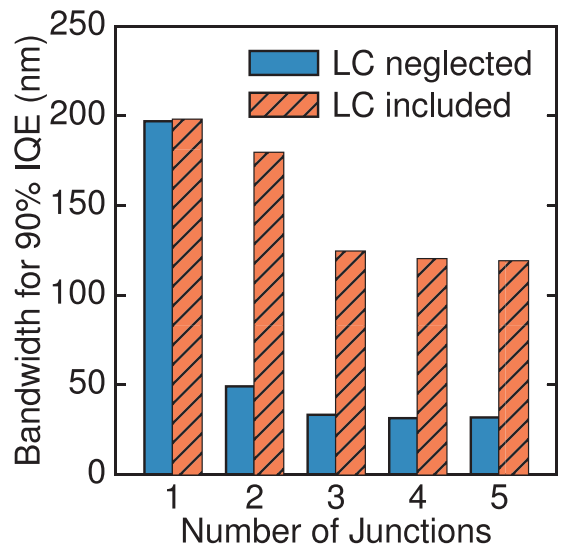

FIG. 1. (a) General structure of the 5junction phototransducer device. Photons are shown being emitted from junction 4 and propagating into junction 5 (luminescent coupling, LC) and being emitted from junction 4 and reabsorbed in the same junction (photon recycling, PR). (b) Simulated internal quantum efficiency bandwidth (at $90 \%$ of peak) for 1- to 5- junction phototransducer devices. Solid blue bars have luminescent coupling neglected, while hatched orange bars have luminescent coupling included in the model. well-known and characterized GaAs-based materials (enabling accurate models), and its five optically inter-coupled junctions exhibiting high internal radiative efficiency. For example, if we consider simulations of a series of devices with one to five junctions, we find that the devices become increasingly sensitive to the illuminating wavelength (Fig. 1(b)). However, the inclusion of luminescent coupling significantly impacts the computed performance, greatly extending the range of usable wavelengths, and demonstrating the importance of including it in the numerical model.

\section{MATERIALS AND METHODS}

The devices discussed here were grown by metalorganic chemical vapour deposition (MOCVD) in an Aixtron 2600 reactor, on $150 \mathrm{~mm}$ (100) p-type GaAs substrates. The devices are $2.4 \mathrm{~mm} \times 2.4 \mathrm{~mm}$ and have a circular aperture of $2.1 \mathrm{~mm}$ diameter (Fig. 2(a)). They were fabricated with a pattern of $5 \mu \mathrm{m}$ wide silver gridlines forming the top contact, and a two-layer anti-reflective coating optimized for transmission at $835 \mathrm{~nm}$.

Efficiency of the fabricated devices was determined from current-voltage $(J-V)$ curves measured while the device was illuminated by a fiber-coupled JDSU L4-2486 laser temperature-tuned to operate at $835 \mathrm{~nm}$, using a Keithley 2420 source-measure unit, Figs. 2(b) and 2(c). Measurements were made under steady-state conditions. Device temperature was regulated at $25^{\circ} \mathrm{C}$, and power output from the laser was calibrated using a Thorlabs S121C silicon photodiode power sensor. Uncertainty in the measured efficiency was estimated at $\pm 5 \%$ (relative) and was primarily limited by modal instability of the illuminating laser.

Internal quantum efficiency of the samples was measured using a Newport IQE-200 system including a chopper with digital lock-in amplifier at $87 \mathrm{~Hz}$ and independent measurement of specular and diffuse reflectivity. The system is calibrated using NIST traceable calibration standards.

The phototransducer devices were simulated using a 2D finite-volume model in the drift-diffusion based device simulator Synopsys Sentaurus using published material parameters, and including realistic parameters for doping-dependent SRH (Shockley-Read-Hall) recombination, Auger recombination, and surface recombination. Radiative recombination parameters are selected with care, as lifetimes derived from typical double heterostructure measurements may be artificially enhanced by photon recycling. Maximum SRH lifetime in GaAs was set at $\tau_{\max }=10^{-4} \mathrm{~s}$ except where otherwise noted, with the lifetime in all layers being reduced as a function of doping according to the Scharfetter relation. ${ }^{15}$ A small interface recombination velocity of $100 \mathrm{~cm} / \mathrm{s}$ was also included at all interfaces between GaAs and the adjacent layers.

The effects of photon recycling and luminescent coupling are modeled using a two-stage process. Prior to running the device simulator, coupling coefficients are calculated relating the radiative recombination rate at each mesh vertex with generation rates at all other vertices. This calculation is discussed in detail in the Appendices. Then, the coupled generation is added as an additional term in the continuity equation via the nonlocal recombination "Physical Model Interface" (PMI) in Sentaurus Device. The PMI is a plug-in mechanism which enables custom code to be used in combination with the standard device solver.

\section{RESULTS}

In cases where luminescent coupling is neglected in the analysis of multi-junction photovoltaic devices, the device short-circuit current density is often treated as being limited by the minimum of the junction current densities, $J_{\mathrm{SC}}=\min \left(J_{\mathrm{SC}, m}\right)$ for $m=1$ to $M$, where $M$ is the number of junctions. For the phototransducer devices presented here, this approach leads to a significant underestimation of quantum efficiency away from the design wavelength. In order to model the behavior more precisely, we modify the continuity equation to incorporate a coupled generation term, $G_{\text {coupled }}$, which is then solved by the device simulator. We now have relations for the divergence of electron and hole carrier concentrations, $n$ and $p$,

$$
\begin{gathered}
\nabla n=G_{\text {ext }}+G_{\text {coupled }}-R_{\mathrm{SRH}}-R_{\text {Auger }}-R_{\text {rad }}, \\
\nabla p=G_{\text {ext }}+G_{\text {coupled }}-R_{\mathrm{SRH}}-R_{\text {Auger }}-R_{\text {rad }},
\end{gathered}
$$

where $G_{\text {ext }}$ is the generation rate due to external illumination, and $R_{\mathrm{SRH}}, R_{\text {Auger }}$, and $R_{\text {rad }}$ refer to rates for $\mathrm{SRH}$, Auger, and 

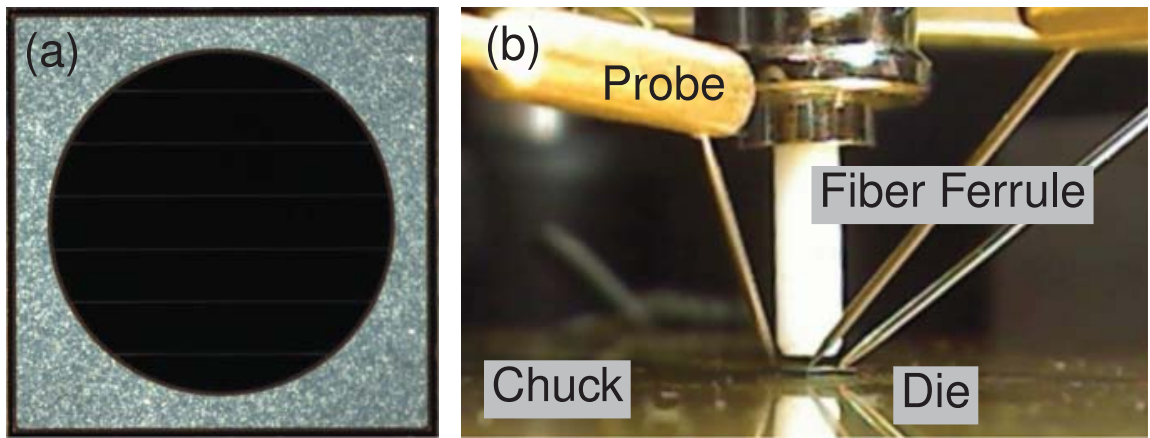

(c)

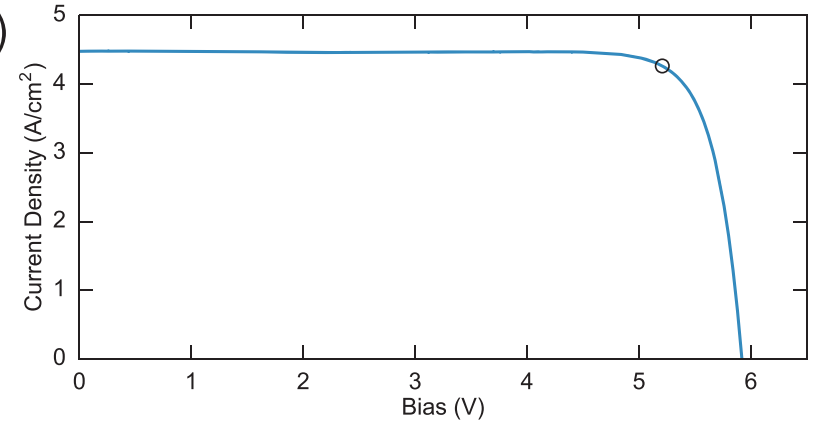

FIG. 2. (a) Top view of a phototransducer die, including the circular aperture with $5 \mu \mathrm{m}$ wide gridlines. (b) Four-wire $J-V$ measurements were completed by contacting the top of the device by two current probes and one voltage-sense probe. The bottom of the device was contacted by the temperature-controlled, gold-plated stage. Illumination is provided by a fiber-coupled laser held at a controlled height above the device. (c) A sample $J$ - $V$ curve under $1.3 \mathrm{~W}, 835 \mathrm{~nm}$ illumination. The small circle indicates the maximum power point. radiative recombination rates, respectively. The coupled generation term at a position $B$ is defined with

$$
G_{\text {coupled }}=\int_{V} K_{B A} R_{\text {rad,A }} d A
$$

where $K_{B A}$ is a coupling factor relating the generation rate at any location $B$ in the device with the radiative recombination rate at a location $A$. This factor is calculated using a transfer matrix method (TMM) where an interface is added at the point of emission within the structure and an internal source term is added as described in the Appendix. The coupled generation is integrated over all locations $A$ within the device volume $V$.

Fig. 3(a) shows an example of the solution of the TMM for a single wavelength, angle of emission, and polarization, with emissions occurring at a selected depth near the top of the fifth GaAs n-p junction. The real parts of the forwardand backward-propagating components of the electric field are shown, along with the magnitude of the total field. In Fig. 3(b), the resulting re-absorbed energy density (fraction of emitted power re-absorbed per unit distance in the $z$ direction) is shown, with a majority of the emissions being reabsorbed in the fifth junction and significant amounts also being coupled into the other junctions. The re-absorbed energy density drops to zero in the window and tunnel junction layers which are composed of higher band gap materials.

The overall coupling coefficient between any given source point and absorption point is a weighted integral of the re-absorbed energy density over all wavelengths, emission angles, and polarizations.

Fig. 3(c) presents the complete coupling matrix $\mathbf{K}$, relating the coupling between a point of emission $z_{\mathrm{A}}$ and a point of re-absorption $z_{\mathrm{B}}$. The squares along the main diagonal indicate the strength of photon recycling (re-absorption of emitted photons within the same material layer where they were emitted). In all cases, the photon recycling efficiency is strong, as indicated by the brightest parts of the map. There is also significant re-absorption in some boxes which lie off of the main diagonal; these represent coupling between different GaAs junctions. It should be noted that Figs. 3(b) and 3 (c) refer only to the rate with which emitted photons are reabsorbed; in order to obtain strong luminescent coupling, there must also be strong radiative emissions. If carriers are being collected much faster than their recombination lifetime or if non-radiative processes dominate, then there will be little coupling even if the coupling coefficient is high.

In order to give a sense of the relative strength of coupling, a simplifying assumption can be made that the radiative recombination rate is constant throughout each junction. In this case, a coupling coefficient linking any two junctions can be calculated by integrating over the depth of the source and destination junctions, shown in Table I. This assumption of uniform emission is not made for the full device simulation which is described below because the solution is handled more exactly. Having calculated the coupling matrix $\mathbf{K}$, we proceed with a complete device simulation with the coupled generation included in the continuity equations, as in Eq. (1). Fig. 4(a) shows the calculated cross-sectional energy band diagram for the 5-junction phototransducer while illuminated with light at its design wavelength $(835 \mathrm{~nm})$ and operating at the maximum-power bias voltage. The conduction band and valence band energies $E_{\mathrm{C}}$ and $E_{\mathrm{V}}$ are shown, along with the electron and hole quasi-Fermi levels $E_{\mathrm{Fn}}$ and $E_{\mathrm{Fp}}$, respectively. In Fig. 4(b), the generation and recombination terms of the continuity equation are plotted as a function of depth within the device. In this case, photogenerated carriers are generated equally and collected efficiently from all junctions so there is minimal recombination, however, the radiative process is dominant compared with the SRH and Auger processes. The radiative emissions are nearly balanced by coupled generation, leading to an 

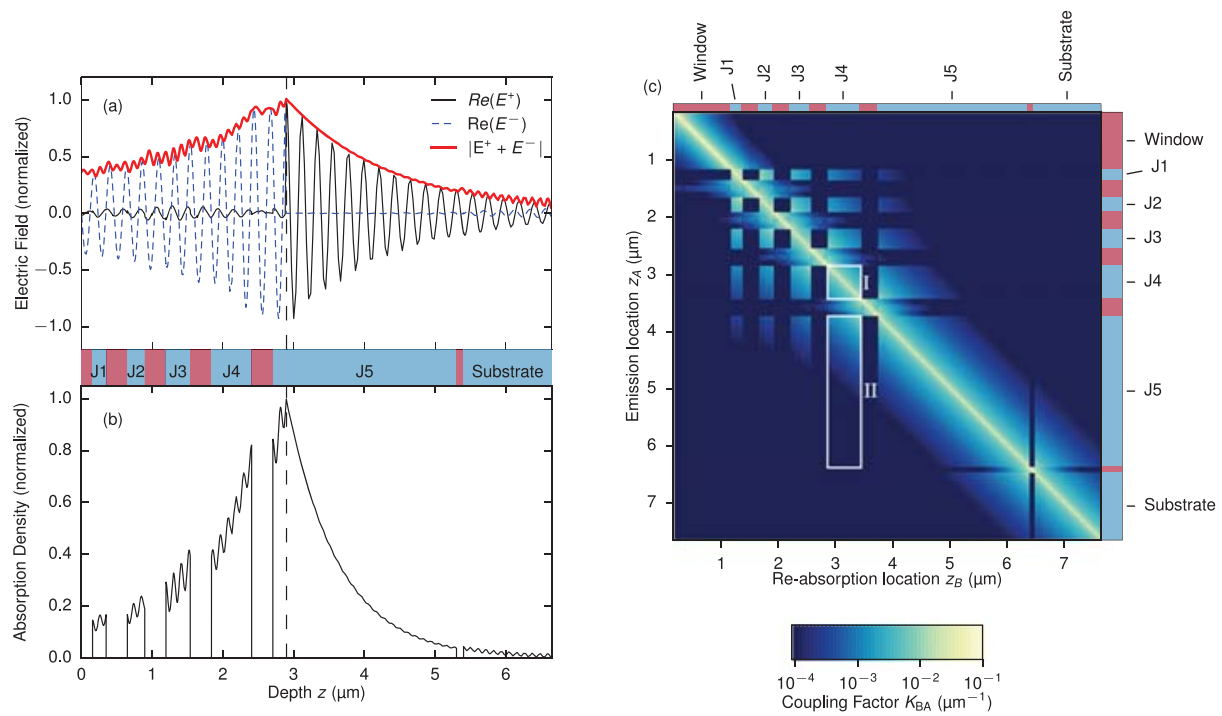

FIG. 3. (a) Example of a TMM calculation for emission at a selected position internal to the structure (indicated by the vertical dashed line) for a single wavelength, angle of propagation, and polarization. The real parts of the forward and backward propagating field components, $E^{+}$and $E^{-}$are shown, along with the magnitude of the total field $\left|E^{+}+E^{-}\right|$. GaAs material layers are indicated with blue shading. The remaining layers, which have a larger band gap, are shaded in red. (b) Re-absorbed energy density as a function of position within the device for the same conditions. (c) Map of the optical coupling matrix, $\mathbf{K}$ for a 5 junction phototransducer device. Boxes along the diagonal indicate the strength of photon recycling, as in region I. Off-diagonal boxes indicate the strength of luminescent coupling between different layers, as in region II.

increase in carrier concentrations and increased separation of the quasi-Fermi levels in each of the junctions.

In Fig. 4(c), the same quantities are plotted for $750 \mathrm{~nm}$ illumination, which is away from the design wavelength of the device. In that case, the external photogenerated current in the bottom junction (J5) is much less than in the other junctions, leading to uncollected photocurrent and large radiative emissions from the top four junctions. There is a net transfer of generated carriers from the top junctions into the bottom one, as evidenced by the $\mathrm{J} 5$ coupled generation that is much greater than the radiative emissions and comparable to the external generation in that region. From examining Fig. 3(c), we can see that it is unlikely that any photon emitted from J1 or J2 would propagate all the way into J5 without being re-absorbed in one of the other GaAs regions, so this transfer relies on a very high internal radiative efficiency and

TABLE I. Coupling coefficients indicate the coupled carrier generation rate in each junction as a fraction of radiative recombination rate in an emitting junction. The last column gives the total coupling efficiency (probability that emitted photons will be re-absorbed in any of the junctions). The highlighted diagonal values represent emission and re-absorption within the same junction.

Junction-to-junction coupling coefficients

\begin{tabular}{cccccccc}
\hline & \multicolumn{5}{c}{ Re-absorbing junction } \\
\cline { 2 - 6 } & & J1 & J2 & J3 & J4 & J5 & Total \\
\hline \multirow{2}{*}{ Emitting junction } & J1 & $\mathbf{0 . 5 1 3}$ & 0.253 & 0.119 & 0.059 & 0.021 & 0.965 \\
& J2 & 0.200 & $\mathbf{0 . 4 4 7}$ & 0.212 & 0.083 & 0.027 & 0.969 \\
& J3 & 0.067 & 0.151 & $\mathbf{0 . 5 0 3}$ & 0.205 & 0.049 & 0.975 \\
& J4 & 0.020 & 0.035 & 0.124 & $\mathbf{0 . 6 1 6}$ & 0.185 & 0.980 \\
& J5 & 0.002 & 0.003 & 0.006 & 0.041 & $\mathbf{0 . 8 8 2}$ & 0.934 \\
\hline \hline
\end{tabular}

good confinement of photons inside the structure to enable multiple successive re-emission and re-absorption events.

We have measured internal quantum efficiency (IQE) of two device samples from different fabrication runs (Fig. 5(a)). Sample 1 is the sample which produced the $61.3 \%$ efficiency and $5.92 \mathrm{~V}$ open-circuit voltage mentioned in the introduction; sample 2 was from an earlier batch and had contacts which were not appropriate for high intensity $J-V$ measurements. The two have similar peaks in their quantum efficiencies at $835 \mathrm{~nm}$, but remarkably different IQE away from the peak despite having nominally the same layer structure. In fact, both samples have a broader IQE than would be expected if current were limited by the junction with lowest externally generated photocurrent (i.e., without luminescent coupling, LC). Fig. 5(a) also shows results of device simulations with two different values for the SRH lifetime, as well as a case where luminescent coupling has been neglected entirely. With luminescent coupling neglected, the IQE is dictated by the collected photocurrent of the bottom junction for wavelengths shorter than $835 \mathrm{~nm}$, and by the collected photocurrent of the top junction for longer wavelengths. This result is independent of lifetime for $\tau_{\max }>10^{-7} \mathrm{~s}$. When luminescent coupling is included in the device simulation, we see a broadening of the IQE peak which closely resembles the broadening of the experimental device measurements. The minor discrepancy in the simulation over the wavelength range of $680-750 \mathrm{~nm}$ may be lessened by incorporating data from optical measurements of the as-grown epitaxial materials (e.g., absorption in the tunnel junctions) and as-deposited antireflection coating layers. Samples 1 and 2 are matched for values of $\tau_{\max }=10^{-6} \mathrm{~s}$ and $\tau_{\max }=10^{-4} \mathrm{~s}$, respectively. In the latter condition, SRH and Auger recombination rates have similar 

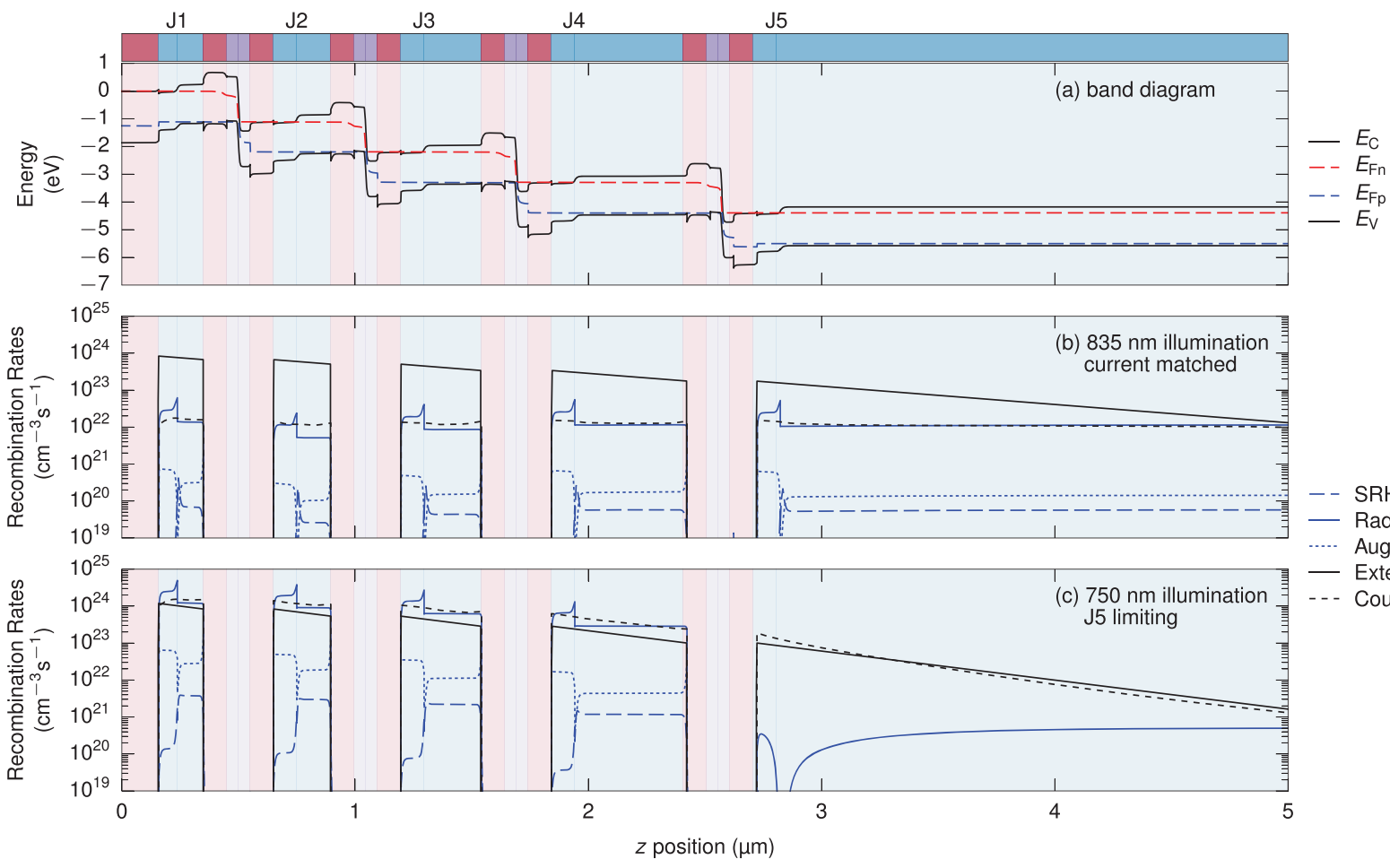

- - SRH Rec.

- Radiative Rec

.... Auger Rec.

- External Gen.

FIG. 4. (a) Calculated energy band diagram of the 5 -junction phototransducer operating at its maximum power point under $835 \mathrm{~nm}$ illumination. Light is incident from the left. The GaAs p-n junctions are indicated with "J1"..."J5" and are shaded in blue; the n++-p++ tunnel junctions are shaded in purple. Generation and recombination terms are shown for the same operating conditions under illumination by (b) the design wavelength (835 nm) and (c) a wavelength causing $\mathrm{J} 5$ to be strongly current limiting $(750 \mathrm{~nm})$.

magnitudes, so further increases in SRH lifetime will not significantly improve performance.

As a metric to discuss the reduction in spectral sensitivity due to luminescent coupling, we consider the spectral width where the IQE exceeds $90 \%$ of its maximum (FW90M). In Fig. 5(a), the FW90M of $31 \mathrm{~nm}$ in the simulation neglecting

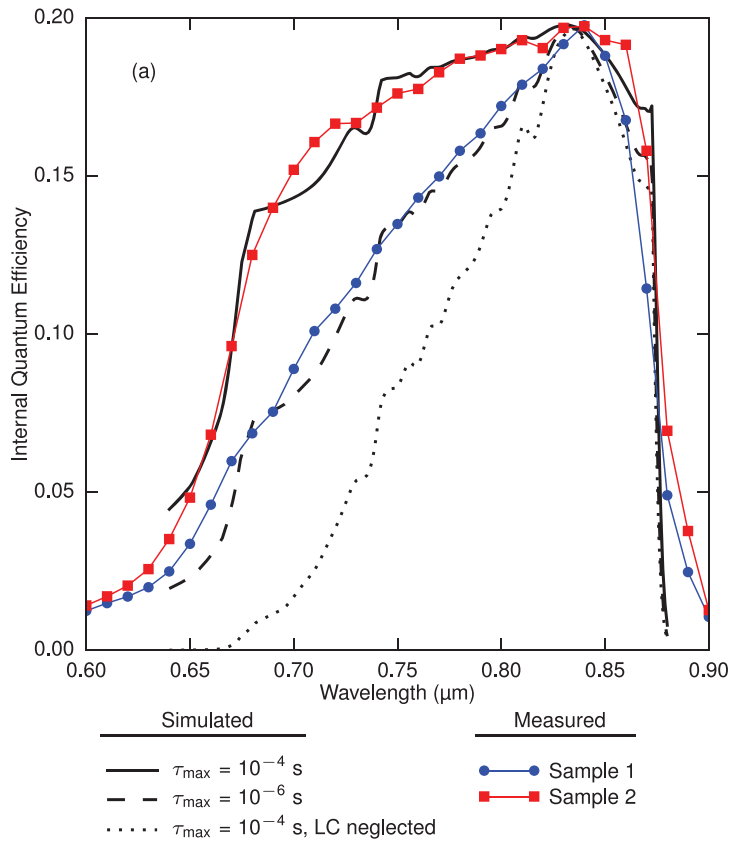

LC is broadened to $104 \mathrm{~nm}$ and $238 \mathrm{~nm}$ in the measurements of samples 1 and 2, respectively.

In addition to the effect on $\mathrm{QE}$, the simulation indicates a benefit to voltage of the device due to photon recycling. As was shown in Table I, there is a $>40 \%$ probability that photons emitted from any of the junctions will be re-absorbed in

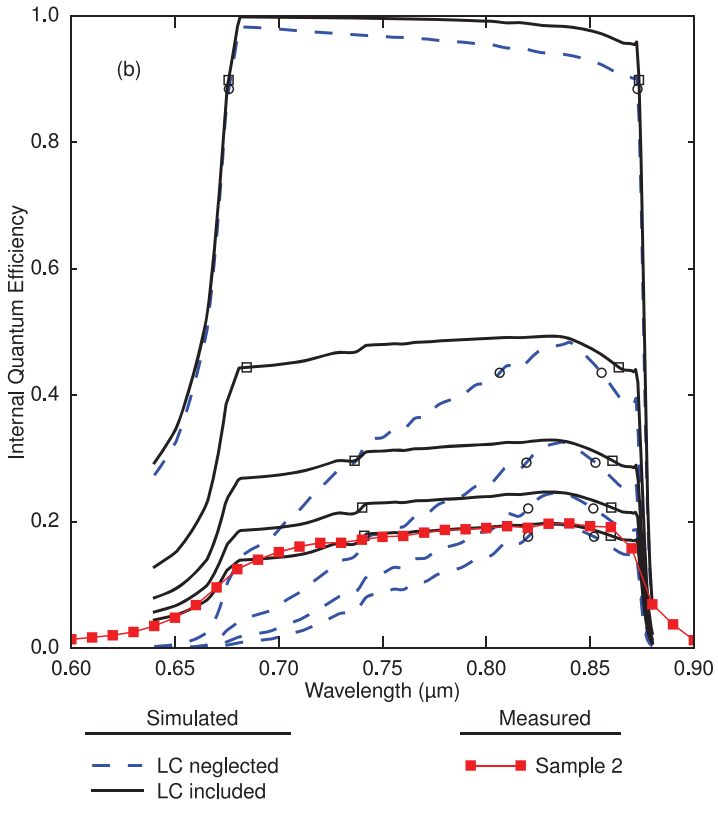

FIG. 5. (a) Simulated and measured IQE of a five-junction phototransducer device. Photocurrent is divided over five junctions, yielding a maximum possible IQE of 0.2. (b) Simulated IQE of designs with one to five junctions. Dashed blue curves neglect luminescent coupling; solid black lines include luminescent coupling. The circles and squares indicate where each curve falls to $90 \%$ of peak. The measured IQE of sample 2 is shown again here for reference. 
the same junction, and $>90 \%$ probability that they are reabsorbed in one of the junctions. Combined with high internal radiative efficiency where $\tau_{\max }=10^{-4} \mathrm{~s}$, this leads to an overall increase in $V_{\text {OC }}$ of $350 \mathrm{mV}$ for the device $(70 \mathrm{mV}$ per junction) under $1 \mathrm{~W}$ illumination compared with the case where luminescent coupling is neglected. Where $\tau_{\max }=10^{-6}$ $\mathrm{s}$, the increase is $90 \mathrm{mV}$ (18 $\mathrm{mV}$ per junction). We showed previously that the $\tau_{\max }=10^{-6} \mathrm{~s}$ case matched well with the IQE of our sample 1; the simulated $V_{\mathrm{OC}}$ is $5.9 \mathrm{~V}$ when we apply an approximate correction for the additional diode area which is shaded by the top busbar, comparable to the measured $5.92 \mathrm{~V}$. These values are similar to what could be expected from high quality single junction GaAs cells without a back reflector, ${ }^{1}$ which also benefit from photon recycling. 6

In the context of multi-junction solar cells, Friedman has found that the effects of photon recycling are relatively small as the coupling is uni-directional: emissions can only proceed from higher to lower band gap junctions and so there is minimal accumulation of carriers (and minimal benefit in terms of voltage) in the high-band-gap junctions. ${ }^{16}$ Here, there is free coupling between all junctions with minimal losses, and so we find a significant voltage increase across all junctions. The fifth junction (J5) has a lower total coupling efficiency than the others, at 0.934 . This is primarily because photons emitted from J5 can couple into the substrate where carriers can diffuse far from the active device layers and be "lost." A back reflecting structure could be helpful to prevent this but was not included in this design.

Some researchers have used internal radiative efficiency as an input parameter to their models; ${ }^{17,18}$ here, the internal radiative recombination is allowed to vary freely within each layer as a function of material parameters and operating conditions, but Fig. 4 shows that it was typically $>90 \%$ in all GaAs layers for the SRH lifetime of $\tau_{\max }=10^{-4} \mathrm{~s}$ which matched the IQE of our sample 1.

Applying the same analysis to simulated devices with increasing numbers of junctions, we find increased spectral sensitivity, but luminescent coupling tends to mitigate this significantly, as shown in Fig. 5(b) and summarized in Fig. 1(b). In each case, the structure is designed to split the external optical generation evenly across all junctions at the specified illumination wavelength. The same broadening of IQE that was observed in the five-junction case is also apparent in two-, three-, and four-junction designs. In the singlejunction design, the thick $(3.85 \mu \mathrm{m})$ base region and a high base doping of $9 \times 10^{17} \mathrm{~cm}^{-3}$ lead to $\sim 5 \%$ loss of quantum efficiency via radiative recombination in the simulation with luminescent coupling neglected. These losses are eliminated when LC is included in the simulation.

\section{CONCLUSIONS}

We have developed a new method for incorporating luminescent coupling and photon recycling phenomena into device simulations by adding a term to the continuity equation. This approach integrates very cleanly into the simulation process, making it possible to study these effects with no additional work required in the setup of the simulation. With the inclusion of luminescent coupling, we find very good agreement with measured five-junction devices in terms of IQE and spectral sensitivity. In general, we find that the simulation method described here is a convenient way of incorporating quantitative modeling of luminescent coupling effects into device simulations. This approach, unlike other published methods, ${ }^{16}$ avoids presupposing how these effects will manifest themselves, for example, in lumped parameter models as a current source term added to a particular junction. These LC effects can significantly impact the operation of photovoltaic devices through increased photovoltage, broadened quantum efficiency, and reduced sensitivity to current matching. In addition to photovoltaics, this technique can be applied to light emitting diodes, lasers, and integrated photonic systems.

\section{ACKNOWLEDGMENTS}

The authors would like to thank CMC Microsystems, Inc. for providing facilities used in this project. The authors also thank the Natural Sciences and Engineering Research Council (NSERC), Ontario Centers of Excellence (OCE), and the Canada Research Chairs program for funding support. D.M. and S.F. are co-founders of Azastra Opto, Inc., which is the provider of the phototransducer device samples tested in this work.

\section{APPENDIX A: ANGLE OF PROPAGATION IN AN ABSORBING MEDIUM}

Consider a plane wave within an absorbing layer of a multi-layer stack, propagating at some angle to the normal. Since the magnitude of the electromagnetic wave must be uniform in the lateral direction, the planes of constant phase and of constant magnitude are in different directions, i.e., we are dealing with a non-uniform plane wave.

We will assume here that the interface normals are oriented in the $\hat{z}$ direction, and the direction of propagation is in the $x-z$ plane, though the same argument will hold for other azimuthal angles. Following a similar line of argument as Orfanidis, ${ }^{19}$ a non-uniform plane wave can be expressed as

$$
\mathbf{E}(\mathbf{r}, t)=\mathbf{E}_{0} e^{j \mathbf{k} \cdot \mathbf{r}} e^{-j \omega t}
$$

where the complex wave vector $\mathbf{k}$ is defined in terms of two real vectors as $\mathbf{k}=\mathbf{k}_{1}+j \mathbf{k}_{2}$. For consistency, the wave equation requires

$$
\begin{gathered}
\mathbf{k} \cdot \mathbf{k}=\left(\mathbf{k}_{1}+j \mathbf{k}_{2}\right)\left(\mathbf{k}_{1}+j \mathbf{k}_{2}\right)=\frac{\left(2 \pi n_{c}\right)^{2}}{\lambda^{2}}, \\
\mathbf{k}_{1} \cdot \mathbf{k}_{1}-\mathbf{k}_{2} \cdot \mathbf{k}_{2}+2 j \mathbf{k}_{1} \cdot \mathbf{k}_{2}=\frac{4 \pi^{2}}{\lambda^{2}}\left(n^{2}-\kappa^{2}+2 j n \kappa\right),
\end{gathered}
$$

where $n_{c}=n+j \kappa$ is the complex index of refraction, and $\lambda$ is the wavelength in vacuum. Equating real and imaginary parts,

$$
\begin{aligned}
\left|\mathbf{k}_{1}\right|^{2}-\left|\mathbf{k}_{2}\right|^{2} & =\frac{4 \pi^{2}}{\lambda^{2}}\left(n^{2}-\kappa^{2}\right), \\
\mathbf{k}_{1} \cdot \mathbf{k}_{2} & =\frac{4 \pi^{2}}{\lambda^{2}} n \kappa .
\end{aligned}
$$


The time-independent part of the plane wave then expands to

$$
\mathbf{E}(\mathbf{r})=\mathbf{E}_{0} e^{j \mathbf{k}_{1} \cdot \mathbf{r}} e^{-\mathbf{k}_{2} \cdot \mathbf{r}}
$$

In an absorbing medium, $\mathbf{k}_{1}$ and $\mathbf{k}_{2}$ may be oriented in different directions, resulting in different normals for the planes of constant phase and planes of constant magnitude. In particular, in a problem which is uniform in the $\hat{\mathbf{x}}$ direction, the $\mathbf{k}_{2}$ vector which defines the attenuation of the wave must be oriented in the direction of the interface normal (assumed to be $\hat{\mathbf{z}}$ ): $\mathbf{k}_{2} \cdot \hat{\mathbf{x}}=0$. For waves propagating in a direction $\theta$ with respect to the $z$ axis, (A1) can be re-arranged as

$$
\left|\mathbf{k}_{1}\right|^{2}=\frac{4 \pi^{2}}{\lambda^{2}}\left(n^{2}-\kappa^{2}\right)+\left|\mathbf{k}_{2}\right|^{2}
$$

Squaring (A2) and substituting (A3),

$$
\begin{gathered}
\left|\mathbf{k}_{1}\right|^{2}\left|\mathbf{k}_{2}\right|^{2} \cos ^{2}(\theta)=\left(\frac{4 \pi^{2}}{\lambda^{2}} n \kappa\right)^{2}, \\
\left|\mathbf{k}_{2}\right|^{4}+\left[\frac{4 \pi^{2}}{\lambda^{2}}\left(n^{2}-\kappa^{2}\right)\right]\left|\mathbf{k}_{2}\right|^{2}-\left(\frac{4 \pi^{2}}{\lambda^{2} \cos ^{2} \theta} n \kappa\right)^{2}=0 \\
\left|\mathbf{k}_{2}\right|^{2}=\frac{-\frac{4 \pi^{2}}{\lambda^{2}}\left(n^{2}-\kappa^{2}\right) \pm \sqrt{\left[\frac{4 \pi^{2}}{\lambda^{2}}\left(n^{2}-\kappa^{2}\right)\right]^{2}+4\left(\frac{4 \pi^{2}}{\lambda^{2} \cos ^{2} \theta} n \kappa\right)^{2}}}{2} .
\end{gathered}
$$

$\left|\mathbf{k}_{2}\right|$ must be real and positive. The vector $\mathbf{k}_{2}$ is then $\left|\mathbf{k}_{2}\right| \hat{z}$. $\left|\mathbf{k}_{1}\right|$ is given by (A3) or (A4), and the vector is in the direction $\theta$. Finally, we note that the complex-valued angle of incidence of a wave with wave vector $\mathbf{k}$ is found from

$$
\begin{gathered}
\mathbf{k} \cdot \hat{z}=|\mathbf{k}| \cos (\zeta), \\
\zeta=\cos ^{-1} \frac{\mathbf{k} \cdot \hat{z}}{|\mathbf{k}|} .
\end{gathered}
$$

For materials with extinction coefficient equal to zero, $\zeta$ will be the same as the angle of propagation $\theta$. But where emissions originate from a material with non-zero extinction coefficient the complex-valued angle $\zeta$ must be used as the angle of incidence in Snell's law and to calculate Fresnel's reflectivity and transmissivity coefficients $r$ and $t$.

\section{APPENDIX B: TMM FOR AN INTERNAL SOURCE}

As noted above, we avoid problems which can arise from interfacing regions of ray optics and regions of wave optics by treating the entire structure, including the emitting layer, with a self-consistent wave optics approach. In order to do this, we adapt the TMM for the case of emissions from a position within a planar layer stack.

In the usual TMM approach, the phasors representing the magnitude of forward- and backward-propagating electric fields at the interfaces of a multi-layer stack are related using matrices (Fig. 6). We refer to propagation from left to right as "forward" and right to left as "backward." The fields at the left and right edges of a layer $i$ are related by a propagation matrix $\mathbf{P}_{i}$

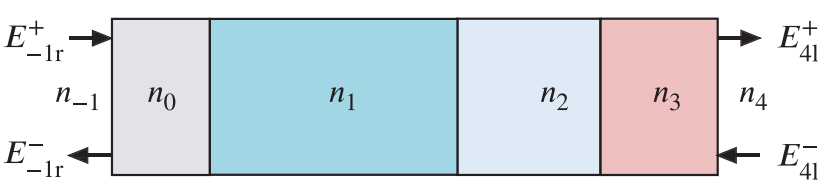

FIG. 6. Example of terminology for the conventional TMM calculation, in the case of a 4-layer material stack.

$$
\left[\begin{array}{c}
E_{i 1}^{+} \\
E_{i \mathrm{l}}^{-}
\end{array}\right]=\left[\begin{array}{cc}
e^{-j k_{z i} d_{i}} & 0 \\
0 & e^{j k_{z i} d_{i}}
\end{array}\right]\left[\begin{array}{c}
E_{i \mathrm{r}}^{+} \\
E_{i \mathrm{r}}^{-}
\end{array}\right], \quad\left[\begin{array}{c}
E_{i 1}^{+} \\
E_{i \mathrm{l}}^{-}
\end{array}\right]=\mathbf{P}_{i}\left[\begin{array}{c}
E_{i \mathrm{r}}^{+} \\
E_{i \mathrm{r}}^{-}
\end{array}\right] .
$$

The subscript $i$ refers to the layer number, and 1 and $r$ refer to the left and right edges of the layer. Hence, $E_{i l}^{+}$is the phasor representing the forward-propagating $z$-component of the field at the left side of layer $i, k_{z i}$ is the $z$-component of the wave vector in layer $i$, and $d_{i}$ is the thickness of layer $i$. The fields on the left and right of an interface are related by

$$
\begin{aligned}
& {\left[\begin{array}{l}
E_{i-1 \mathrm{r}}^{+} \\
E_{i-1 \mathrm{r}}^{-}
\end{array}\right]=1 / t_{i-1, i}\left[\begin{array}{cc}
1 & r_{i-1, i} \\
r_{i-1, i} & 1
\end{array}\right]\left[\begin{array}{l}
E_{i 1}^{+} \\
E_{i 1}^{-}
\end{array}\right]} \\
& {\left[\begin{array}{l}
E_{i-1 \mathrm{r}}^{+} \\
E_{i-1 \mathrm{r}}^{-}
\end{array}\right]=\mathbf{D}_{i-1, i}\left[\begin{array}{l}
E_{i 1}^{+} \\
E_{i 1}^{-}
\end{array}\right],}
\end{aligned}
$$

where $r_{i-1, i}$ and $t_{i-1, i}$ are the Fresnel reflection and transmission coefficients between layers $i-1$ and $i .{ }^{19}$ As an example, consider the layer stack in Fig. 6. The fields at the far left and right edges of the stack are related by

$$
\left[\begin{array}{l}
E_{-1 \mathrm{r}}^{+} \\
E_{-1 \mathrm{r}}^{-}
\end{array}\right]=\mathbf{D}_{-1,0} \mathbf{P}_{0} \mathbf{D}_{0,1} \mathbf{P}_{1} \mathbf{D}_{1,2} \mathbf{P}_{2} \mathbf{D}_{2,3} \mathbf{P}_{3} \mathbf{D}_{3,4}\left[\begin{array}{c}
E_{4 \mathrm{l}}^{+} \\
E_{4 \mathrm{l}}^{-}
\end{array}\right] \text {. }
$$

Now let us look at a case where a wave is being emitted from a position $z_{\mathrm{A}}$ within layer $i=1$ (Fig. 7). We split the propagation matrix $\mathbf{P}_{i}$ into matrices for the left and right side of $z_{\mathrm{A}}$, and relate fields immediately to the left and right side of the newly created source interface as

$$
\begin{aligned}
& {\left[\begin{array}{l}
E_{\mathrm{Al}}^{+} \\
E_{\mathrm{Al}}^{-}
\end{array}\right]=\left[\begin{array}{c}
E_{\mathrm{Ar}}^{+} \\
E_{\mathrm{Ar}}^{-}
\end{array}\right]-\left[\begin{array}{c}
E_{\mathrm{s}} \\
0
\end{array}\right], \quad 0 \leq \theta<\pi / 2,} \\
& {\left[\begin{array}{l}
E_{\mathrm{Al}}^{+} \\
E_{\mathrm{Al}}^{-}
\end{array}\right]=\left[\begin{array}{l}
E_{\mathrm{Ar}}^{+} \\
E_{\mathrm{Ar}}^{-}
\end{array}\right]-\left[\begin{array}{c}
0 \\
E_{\mathrm{s}}
\end{array}\right], \quad \pi / 2 \leq \theta \leq \pi,}
\end{aligned}
$$

where $E_{\mathrm{s}}$ is the strength of the field source at $z_{\mathrm{A}}$, and 1 and $\mathrm{r}$ refer to the left and right sides of the source interface, respectively.

We can solve for the fields emitted from the left and right sides of the stack, and then use those values to find the field at an arbitrary location in the stack. For the portion to the left of the source, we write

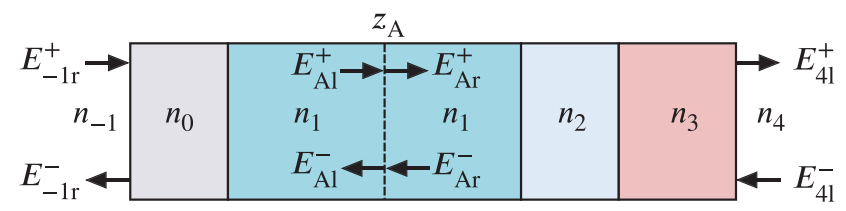

FIG. 7. Nomenclature for the internal-source TMM calculation, with emissions from a depth $z_{\mathrm{A}}$ within layer 1 . 


$$
\left[\begin{array}{l}
E_{-1 \mathrm{r}}^{+} \\
E_{-1 \mathrm{r}}^{-}
\end{array}\right]=\mathbf{D}_{-1,0} \mathbf{P}_{0} \mathbf{D}_{0,1}\left[\begin{array}{cc}
e^{-j k_{y i} z_{\mathrm{A}}} & 0 \\
0 & e^{j k_{z i} z_{\mathrm{A}}}
\end{array}\right]\left[\begin{array}{c}
E_{\mathrm{Al}}^{+} \\
E_{\mathrm{Al}}^{-}
\end{array}\right]=\mathbf{L}\left[\begin{array}{c}
E_{\mathrm{Al}}^{+} \\
E_{\mathrm{Al}}^{-}
\end{array}\right]
$$

where we have defined $\mathbf{L}$ to contain all of the matrices to the left of the source interface. With no wave incident on the left side of the stack, $E_{-1 r}^{+}=0$

$$
\begin{aligned}
{\left[\begin{array}{c}
0 \\
E_{-1 \mathrm{r}}^{-}
\end{array}\right] } & =\left[\begin{array}{ll}
L_{11} & L_{21} \\
L_{12} & L_{22}
\end{array}\right]\left[\begin{array}{c}
E_{\mathrm{Al}}^{+} \\
E_{\mathrm{Al}}^{-}
\end{array}\right], \\
0 & =L_{11} E_{\mathrm{Al}}^{+}+L_{12} E_{\mathrm{Al}}^{-}, \\
\frac{E_{\mathrm{Al}}^{+}}{E_{\mathrm{Al}}^{-}} & =\frac{-L_{12}}{L_{11}} .
\end{aligned}
$$

Similarly for the right side,

$$
\begin{aligned}
{\left[\begin{array}{c}
E_{\mathrm{Ar}}^{+} \\
E_{\mathrm{Ar}}^{-}
\end{array}\right] } & =\left[\begin{array}{cc}
e^{-j k_{z i}\left(d_{i}-z_{\mathrm{A}}\right)} & 0 \\
0 & e^{j k_{z i}\left(d_{i}-z_{\mathrm{A}}\right)}
\end{array}\right] \mathbf{D}_{1,2} \mathbf{P}_{2} \mathbf{D}_{2,3} \mathbf{P}_{3} \mathbf{D}_{3,4}\left[\begin{array}{c}
E_{4 \mathrm{l}}^{+} \\
E_{4 \mathrm{l}}^{-}
\end{array}\right] \\
& =\mathbf{N}\left[\begin{array}{c}
E_{4 \mathrm{l}}^{+} \\
E_{4 \mathrm{l}}^{-}
\end{array}\right] .
\end{aligned}
$$

And with $E_{41}^{-}=0$,

$$
\begin{aligned}
{\left[\begin{array}{c}
E_{\mathrm{Ar}}^{+} \\
E_{\mathrm{Ar}}^{-}
\end{array}\right] } & =\left[\begin{array}{ll}
N_{11} & N_{21} \\
N_{12} & N_{22}
\end{array}\right]\left[\begin{array}{c}
E_{41}^{+} \\
0
\end{array}\right], \\
\frac{E_{\mathrm{Ar}}^{+}}{E_{\mathrm{Ar}}^{-}} & =\frac{N_{11}}{N_{21}} .
\end{aligned}
$$

Applying (B1) for a forward-propagating wave,

$$
E_{\mathrm{Al}}^{+}+E_{\mathrm{s}}=\frac{N_{11}}{N_{21}} E_{\mathrm{Al}}^{-}
$$

and then substituting (B3),

$$
\begin{aligned}
\frac{-L_{12}}{L_{11}} E_{\mathrm{Al}}^{-}+E_{\mathrm{s}} & =\frac{N_{11}}{N_{21}} E_{\mathrm{Al}}^{-}, \\
\frac{L_{12}}{L_{11}} E_{\mathrm{Al}}^{-}+\frac{N_{11}}{N_{21}} E_{\mathrm{Al}}^{-} & =E_{\mathrm{s}}, \\
\left(L_{12} N_{21}+L_{11} N_{11}\right) E_{\mathrm{Al}}^{-} & =L_{11} N_{21} E_{\mathrm{s}} .
\end{aligned}
$$

Finally, we arrive at

$$
\begin{gathered}
E_{\mathrm{Al}}^{-}=\frac{L_{11} N_{21}}{L_{12} N_{21}+L_{11} N_{11}} E_{\mathrm{s}}, \text { and } \\
E_{\mathrm{Al}}^{+}=\frac{-L_{12} N_{21}}{L_{12} N_{21}+L_{11} N_{11}} E_{\mathrm{s}} .
\end{gathered}
$$

These last expressions (B5) and (B6) can be computed directly by assuming a value of unity for $E_{\mathrm{s}}$, and from there the forward and backward field components on left and right sides of the source can be found readily. The $\mathbf{P}_{i}$ and $\mathbf{D}_{i}$ matrices can then be used to determine the electric field components at arbitrary positions throughout the stack. Similarly for a backward propagating wave, we can find that

$$
\begin{aligned}
& E_{\mathrm{Al}}^{-}=\frac{-L_{11} N_{11}}{L_{11} N_{11}+L_{12} N_{21}} E_{\mathrm{s}}, \\
& E_{\mathrm{Al}}^{+}=\frac{L_{12} N_{11}}{L_{11} N_{11}+L_{12} N_{21}} E_{\mathrm{s}} .
\end{aligned}
$$

It should be noted that the transfer matrix method can suffer from issues with numerical precision in problems with highly absorbing layers. These result in matrix elements with very large and small magnitudes. Often, this problem can be addressed by recognizing that a particular layer will (in effect) completely absorb the wave entering it from the source. This layer can then be treated as a "semi-infinite" layer bounding the stack on that side, and so the $\mathbf{P}_{i}$ matrix representing propagation through that layer (and others beyond it) can be omitted from the process of assembling the $\mathbf{L}$ and $\mathbf{N}$ matrices. Typically, this decision must be made separately for every wavelength and emission angle. The TMM calculation has also been implemented using 256-bit complex floating-point numbers, i.e., each complex number is represented by a 128-bit real part and a 128-bit imaginary part. However, this on its own is not sufficient to provide an accurate solution in the case of a thick absorbing substrate.

\section{APPENDIX C: RE-ABSORBED ENERGY DENSITY AT A GIVEN POSITION}

In order to find the re-absorbed energy density (fraction of the emitted energy absorbed per unit distance) at a given position $z_{\mathrm{B}}$, we first find a solution of the TMM as described in Appendix B and evaluate the forward and backward components of the electric field, $E^{+}$and $E^{-}$at $z_{\mathrm{B}}$. The Poynting vector, $\mathbf{S}$ can also be evaluated at $z_{\mathrm{B}}$, and the negative derivative of the Poynting vector gives the re-absorbed energy density. We use the same method described by Byrnes. ${ }^{20}$

\section{TE polarization}

Following Byrnes, we begin by writing out the electric and magnetic fields $\mathbf{E}$ and $\mathbf{H}$ at the position of interest

$$
\begin{aligned}
\mathbf{E}= & E^{+} \hat{\mathbf{y}}+E^{-} \hat{\mathbf{y}} \\
\mathbf{H} \propto & n_{\mathrm{c}} E^{+}(-\cos \zeta \hat{\mathbf{x}}+\sin \zeta \hat{\mathbf{z}}) \\
& +n_{\mathrm{c}} E^{-}(\cos \zeta \hat{\mathbf{x}}+\sin \zeta \hat{\mathbf{z}}) \\
\mathbf{S} \cdot \hat{\mathbf{z}}= & \frac{1}{2} \operatorname{Re}\left[\hat{\mathbf{z}} \cdot\left(\mathbf{E}^{*} \times \mathbf{H}\right)\right] \\
\propto & \operatorname{Re}\left[\left(E^{+*}+E^{-*}\right)\left(E^{+}-E^{-}\right) n_{\mathrm{c}} \cos \zeta\right] .
\end{aligned}
$$

We evaluate the power emitted from the source using the $\hat{z}$ component of the Poynting vector, $\mathbf{S} \cdot \hat{z}$, evaluated immediately to the left and right of the source interface. The power density emitted by the source is

$$
S_{\text {in }}=\left(\mathbf{S}_{\mathrm{Ar}}-\mathbf{S}_{\mathrm{Al}}\right) \cdot \hat{\mathbf{z}} \text {. }
$$

This result will be used to scale each of the left-and rightpropagating solutions to the required emitted power.

The negative derivative of $\mathbf{S} \cdot \hat{\mathbf{z}}$ is the absorbed power per unit distance in the $z$ direction. Again following Byrnes, we normalize this for unit input power density 


$$
a \equiv \frac{1}{P_{\text {in }}} \frac{d}{d z}(\mathbf{S} \cdot \hat{\mathbf{z}})=\left|E^{+}+E^{-}\right|^{2} \operatorname{Im}\left(n_{\mathrm{c}} k_{z} \cos \zeta\right) / S_{\text {in }} .
$$

We refer to this quantity $a$ as the "absorbed energy density," with units of inverse distance.

\section{TM polarization}

Similarly for TM polarization,

$$
\mathbf{S} \cdot \hat{\mathbf{z}} \propto \operatorname{Re}\left[\left(E^{+}+E^{-}\right)\left(E^{+*}-E^{-*}\right) n_{\mathrm{c}} \cos \zeta^{*}\right]
$$

and

$$
a=\operatorname{Im}\left[n_{\mathrm{c}} \cos \zeta^{*}\left(k_{z}\left|E^{+}-E^{-}\right|^{2}-k_{z}^{*}\left|E^{+}+E^{-}\right|^{2}\right)\right] / S_{\text {in }} .
$$

\section{APPENDIX D: GENERATION DUE TO COUPLING}

Consider a photon emission from point $\mathrm{A}$ in layer $i$, at position $z_{\mathrm{A}}$. Assuming isotropic emission, the rate of photon emission in a direction $(\theta, \phi)$, where $\theta$ is the polar angle relative to the surface normal and $\phi$ is the azimuthal angle, will be $1 / 4 \pi R_{\mathrm{A}}$ per steradian, and will be evenly split between TE and TM polarizations. $R_{\mathrm{A}}$ is the radiative recombination rate, which can be calculated from the electron and hole densities $n$ and $p$, with $R=B_{\text {rad }}\left(n p-n_{\mathrm{i}, \text { eff }}^{2}\right)$. $n_{\mathrm{i}, \text { eff }}$ is the effective intrinsic carrier concentration in the emitting layer. The normalized energy distribution of the emissions is ${ }^{17}$

$$
\hat{S}(E) \approx \frac{\alpha(E) n_{\mathrm{c}}^{2}(E) E^{2} \exp (-E / k T)}{\int_{0}^{\infty} \alpha(E) n_{\mathrm{c}}^{2}(E) E^{2} \exp (-E / k T) d E} .
$$

Let us consider the coupled generation at a point of absorption, B. In order to avoid treating the full complexity of a 2D mesh, we will assume photons are redistributed and re-absorbed uniformly in the lateral direction; this is reasonable if a device's gridlines are small compared with either the carrier diffusion lengths or with the optical absorption lengths, and if the device is uniformly illuminated. We have already shown the absorbed energy density $a(\theta, \phi, E)$ giving the fraction of emissions from a location $z_{\mathrm{A}}$ that will be re-absorbed per unit thickness in a thin layer at $z_{\mathrm{B}}$. Then we can write

$$
G_{\mathrm{B}}(\theta, \phi, E) W=a(\theta, \phi, E) \hat{S}_{\mathrm{A}}(E) R_{\mathrm{A}} V_{\mathrm{A}}
$$

to represent the optical generation rate at $\mathrm{B}$ originating from recombination in a small volume (i.e., a mesh element) $V$ at position A. The variable $W$ refers to the surface area of the device being simulated. This will be integrated over $\theta, \phi$, and $E$ and summed over TE and TM polarizations to get the total optical coupling factor $K_{\mathrm{BA}}$ between mesh vertices at A and B

$$
\begin{aligned}
\frac{G_{\mathrm{B}}}{R_{\mathrm{A}}}= & \frac{1}{8 \pi W} \iint_{0}^{\infty} \int_{0}^{\pi 2 \pi}\left[a_{0}[\theta, \phi, E)+a_{\mathrm{TM}}(\theta, \phi, E)\right] \hat{S}_{\mathrm{A}}(E) V_{\mathrm{A}} d \phi \\
& \times \sin (\theta) d \theta d E
\end{aligned}
$$

$$
\begin{aligned}
K_{\mathrm{BA}} \equiv & \frac{G_{\mathrm{B}}}{R_{\mathrm{A}} V_{\mathrm{A}}}=\frac{1}{4 W} \int_{0}^{\infty} \int_{0}^{\pi}\left[a_{\mathrm{TE}}(\theta, \phi, E)+a_{\mathrm{TM}}(\theta, \phi, E)\right] \\
& \times \sin (\theta) d \theta \hat{S}_{\mathrm{A}}(E) d E .
\end{aligned}
$$

Here, the limits of integration in energy are given as $(0, \infty)$, but in fact $\hat{S}_{\mathrm{A}}(E)$ only holds a significant value over a range of a few $k T$ above and below the emitting band gap and this can be used to reduce the limits of integration in $E$. Prior to running the device simulator, we calculate the square optical coupling matrix, $\mathbf{K}$ containing coupling coefficients $K_{\mathrm{BA}}$ for all combinations of $\mathrm{A}$ and $\mathrm{B}$. In theory, for a mesh with $N$ vertices the matrix would be of size $N \times N$, but we can omit coupling coefficients for layer combinations with minimal coupling, for example, emissions from a layer $i$ to a layer $j$ with

$$
\int_{0}^{\infty} \alpha_{j}(E) \hat{S}_{i}(E) d E \ll 1 / d_{j},
$$

with $\alpha_{j}$ being the absorption coefficient in layer $j, d_{j}$ being the thickness of layer $j$, and $\hat{S}_{i}(E)$ being the luminescent emission spectrum in layer $i$. It is likely that many coefficients will be very small, and so it should be possible to omit many coefficients with minimal effect on the accuracy of the calculation.

\section{APPENDIX E: INTEGRATION WITH THE DEVICE SIMULATOR}

The model is integrated into Senaturus using the "nonlocal generation-recombination" physical model interface (PMI). ${ }^{21}$ This is implemented as a $\mathrm{C}++$ class which is compiled into a shared object file and loaded by Senaturus when needed. The PMI code defines a class which performs several functions: As noted earlier, the radiative recombination rate at vertex $\mathrm{B}$ is given by

$$
R_{\mathrm{B}}=B_{\mathrm{rad}, \mathrm{B}}\left(n p-n_{\mathrm{i}, \mathrm{eff}}^{2}\right) .
$$

The optical generation rate at B due to optical coupling is

$$
G_{\text {coupled }}=\sum_{A=0}^{N} K_{\mathrm{BA}} B_{\text {rad }, \mathrm{A}}\left(n_{\mathrm{A}} p_{\mathrm{A}}-n_{\mathrm{i}, \mathrm{eff}, \mathrm{A}}^{2}\right) V_{A} .
$$

And so the net recombination current due to radiative recombination and optically coupled generation is

$$
R_{\text {elec }, \mathrm{B}}=R_{\text {hole }, \mathrm{B}}=R_{\text {rad }, \mathrm{B}}-G_{\text {coupled, } \mathrm{B}} .
$$

The optical coupling PMI must also provide Jacobian matrices for the dependencies of the electron and hole recombination rates. In this model, the dependencies are the electron and hole densities, so four Jacobian matrices can be provided

$$
\begin{gathered}
\frac{d R_{\text {elec }, \mathrm{B}}}{d n_{\mathrm{A}}}=\frac{d R_{\text {hole }, \mathrm{B}}}{d n_{\mathrm{A}}}=\delta_{\mathrm{A}, \mathrm{B}} B_{\mathrm{rad}, \mathrm{B}} p_{\mathrm{B}}-\sum_{i=0}^{N} K_{\mathrm{BA}} B_{\mathrm{rad}, \mathrm{A}} p_{\mathrm{A}} V_{\mathrm{A}}, \\
\frac{d R_{\text {elec } \mathrm{B}}}{d p_{\mathrm{A}}}=\frac{d R_{\text {hole }, \mathrm{B}}}{d p_{\mathrm{A}}}=\delta_{\mathrm{A}, \mathrm{B}} B_{\mathrm{rad}, \mathrm{B}} n_{\mathrm{B}}-\sum_{i=0}^{N} K_{\mathrm{BA}} B_{\mathrm{rad}, \mathrm{A}} n_{\mathrm{A}} V_{\mathrm{A}} .
\end{gathered}
$$


The method described above can significantly increase the computational cost of a simulation. In the implementation discussed here, several steps can be taken to minimize this cost: (1) the coupling coefficients are calculated only once for any given combination of $z_{\mathrm{A}}$ and $z_{\mathrm{B}}$, which is particularly efficient if the mesh is well structured; (2) the calculation of coupling coefficients is done in parallel, using all available processors; (3) the Sentaurus device simulation is also executed in parallel, with each device region being handled by a separate thread; and (4) in the case of steady-state simulations, the offdiagonal elements of the Jacobians are omitted. This last measure vastly reduces memory requirements and the size of the matrices which must be handled by the non-linear solver. The off-diagonal elements of the Jacobians cannot be omitted in the case of transient (time-dependent) simulations.

${ }^{1}$ M. A. Steiner, J. F. Geisz, D. J. Friedman, A. Duda, W. J. Olavarria, M. Young, D. Kuciauskas, and S. R. Kurtz, IEEE J. Photovolt. 3, 1437 (2013).

${ }^{2}$ D. J. Friedman, J. F. Geisz, and M. A. Steiner, IEEE J. Photovolt. 4, 986 (2014).

${ }^{3}$ M. A. Steiner, J. F. Geisz, T. E. Moriarty, R. M. France, W. E. McMahon, J. M. Olson, S. R. Kurtz, and D. J. Friedman, IEEE J. Photovolt. 3, 879 (2013).

${ }^{4}$ J. Jia, F. Suarez, T. Bilir, V. Sabnis, and J. Harris, AIP Conf. Proc. 1616, 3-7 (2014).

${ }^{5} \mathrm{G}$. Létay, M. Breselge, and A. W. Bett, in 3rd World Conference on Photovoltaic Energy Conversion (2003), pp. 741-744.
${ }^{6}$ G. Létay, M. Hermle, and A. W. Bett, Prog. Photovolt.: Res. Appl. 14, 683 (2006).

${ }^{7}$ A. W. Walker, O. Hohn, D. N. Micha, L. Wagner, H. Helmers, A. W. Bett, and F. Dimroth, Proc. SPIE 9358, 11-13 (2015).

${ }^{8}$ J. L. Balenzategui and A. Martí, Sol. Energy Mater. Sol. Cells 90, 1068 (2006).

${ }^{9}$ W.-C. Ng and G. Letay, Proc. SPIE 6486, 1-10 (2007).

${ }^{10}$ M. M. Wilkins, A. M. Gabr, A. H. Trojnar, H. Schriemer, and K. Hinzer, in Proceedings of 40th Photovoltaics Specialist Conference (Denver, CO, 2014), Vol. 1, pp. 6-9.

${ }^{11}$ M. Wilkins, A. G. Gabr, P. Sharma, H. P. Schriemer, S. Fafard, and K. Hinzer, in Proceedings of 29th EU PVSEC (Amsterdam, NL, 2014).

${ }^{12}$ J. Piprek, Semiconductor Optoelectronic Devices: Introduction to Physics and Simulation (Academic Press, San Diego, CA, 2003), p. 279.

${ }^{13}$ Optoelectronic Devices: Advanced Simulation and Analysis, edited by J. Piprek (Springer, New York, 2006), p. 452.

${ }^{14}$ C. E. Valdivia, M. M. Wilkins, B. Bouzazi, A. Jaouad, V. Aimez, R. Arès, D. P. Masson, S. Fafard, and K. Hinzer, Proc. SPIE 9358, 93580E (2015).

${ }^{15}$ J. Fossum, R. Mertens, D. Lee, and J. Nijs, Solid State Electron. 26, 569 (1983).

${ }^{16}$ D. J. Friedman, J. F. Geisz, and M. A. Steiner, IEEE J. Photovolt. 3, 1429 (2013).

${ }^{17}$ M. A. Steiner, J. F. Geisz, I. García, D. J. Friedman, A. Duda, and S. R. Kurtz, J. Appl. Phys. 113, 123109 (2013).

${ }^{18}$ O. D. Miller, E. Yablonovitch, and S. R. Kurtz, IEEE J. Photovolt. 2, 303 (2012).

${ }^{19} \mathrm{~S}$. Orfanidis, Electromagnetic Waves and Antennas (Rutgers University, 2010), p. 1043.

${ }^{20}$ S. J. Byrnes, TMM Library Manual v0.1.2, Technical Report, 2012.

${ }^{21}$ Sentaurus Device User Guide, vF-2011 (Synopsys, Inc., 2011), p. 1334. 